\title{
Drawing Nice Projections of Objects in Space*
}

\author{
Prosenjit Bose \\ University of British Columbia \\ Vancouver, British Columbia, Canada \\ Pedro Ramos \\ Universidad Politecnica de Madrid \\ Madrid, Spain
}

\author{
Francisco Gomez \\ Universidad Politecnica de Madrid \\ Madrid, Spain \\ Godfried Toussaint \\ McGill University \\ Montreal, Quebec, Canada
}

\section{Introduction}

In our world we are frequently concerned with describing and analyzing three-dimensional (3-D) rigid objects in 3-D space. However, we often have at our disposal only a 2-D medium, such as paper or a computer-graphics screen, on which to display a necessarily incomplete representation or picture of the objects we are interested in. Therefore it is desirable to obtain 2-D representations of our objects that approximate the real objects as faithfully as possible in some sense [KK93], [Ga95]. A sub-field of visualization closely related to the class of problems considered here is graph-drawing [DETT]. One of the archetypal problems in graph-drawing consists of asking, for a given graph, a "nice" drawing of it. A graph in this context is not a rigid object in 3-D space but a more abstract topological structure which permits the shortening, lengthening and bending of its edges to achieve the desired goal. By contrast, we are concerned with rigid metrical objects in 3-D space which are composed of points (vertices) and line segments (edges) and we would like to obtain "nice" projections of these objects on some plane that will afford them.

We are concerned here with parallel or orthogonal projections [FDFH] rather than perspective projections. Parallel projections may be considered as perspective projections in the limit as the view point approaches a location infinitely far away from the object being viewed. Intuitively, we may think of our object as a wire-frame sitting in 3-D space above the horizontal $(x y)$ plane, and the parallel projection of the object on the $x y$-plane as the shadow cast by the wire frame when a light source shines from a point infinitely high along the positive $z$-axis. Obtaining "nice" parallel projections of an object then reduces to the problem of finding a suitable 3-D rotation for the object such that its shadow on the $x y$ plane contains the desired properties.

To date such problems have received scant attention in the computational geometry literature. When the objects are convex polyhedra (solid bodies) several questions have been explored. For example, a problem of interest in robotics concerns the determination of whether a convex polyhedron may be translated through a "door" that has the shape of a convex polygon. Geometrically this problem reduces to determining if the polyhedron has a shadow that fits in the door [St82], [To85]. Algorithms have also been found for determining the projections of a convex polyhedron that minimize or maximize the area of the shadow that the polyhedron makes on a plane when placing a light source at infinity [MS85], [BGK95]. In computer graphics, good projections for radiosity computation are those that yield the most number of facets visible from the viewpoint [Co90]. On the other

* Research of the first author was supported by an NSERC \& Killam Fellowship. Research of the second and third authors was carried out during their visit to McGill University in 1995 and was self-supported. The fourth author was supported by NSERC Grant no. OGP0009293 and FCAR Grant no. 93-ER-0291. 
hand, when the objects are 3-D polygonal objects (skeletons or wire-frames) very little is known. Hirata et al., [HMTT] give bounds on the worst-case combinatorial complexity of the simplest projections of the skeletons of 3-D convex subdivisions onto a plane. Such simple projections have application to the design of efficient 3-D point location query algorithms [PT92]. Closer in spirit to the work presented here, Kamada and Kawai [KK88] present an $O\left(n^{6} \log n\right)$ time algorithm for computing the projection of a wire-frame, that in a sense maximizes the projected minimum distance between parallel segments. Finally, Bhattacharya and Rosenfeld [BR94] have studied a special class of orthographic projections called Wirtinger projections for 3-D polygons.

In the work presented here the objects considered are polygonal structures in 3-D. Such objects include sets of disjoint line segments, 3-D simple polygons, knots, trees, and more generally, sets of segments in which the segments may touch each other at their end points, such as skeletons of 3-D Voronoi diagrams or other subdivisions such as those in [HMTT]. There are many specific geometrical characteristics of the vague notion of the "niceness" of a projective drawing of an object. Some of these are more desirable than others depending on the application in mind. One requirement of "nice" is that all the significant features of the 3-D object should be visible in the projection. In other words, no vertex should lie behind another, no edge should look like a vertex and no edge should hide another edge. Furthermore, no three edges may have an interior point in common. This type of projection, closely related to Wirtinger projections [BR94], is useful in visualizing knots, and in knot theory is called a regular projection [Re83], [Li93]. Another requirement for effective visualization is simplicity. One measure of simplicity is the number of crossings of edges in the projection. It is desirable to obtain the projection that minimizes the number of crossings. We will refer to such projections as minimum-crossing projections. If the minimum number of crossings is zero we call such projections crossing-free. In some applications we may have a 3-D directed tree as an object of interest. Such a tree may represent a system of veins in the human brain for example, where the direction of an edge represents the direction of blood flow in the corresponding vein segment. Here it is of interest to determine if there exists a projection such that all the directions of the edges of the tree are monotonically increasing in a specified direction on the projection plane. In general we call such projections monotonic projections. More specifically, a projection is monotonic if the projected image on the projection plane is monotonic. A planar polygonal chain is monotonic if there exists a direction such that every line orthogonal to this direction, that intersects the chain, yields a point as the intersection. A planar polygon is monotonic if it can be partitioned into two chains each of which is monotonic with respect to the same direction. A tree is monotonic if it contains a root and a direction such that all paths from the root to the leaves are monotonic with respect to that direction. In this paper we investigate the above four types of projections for objects which are sets of disjoint line segments, simple polygons, polygonal chains and trees.

We should add here that the notions of minimum crossing drawings and monotonic drawings are classic visualization problems that have been well studied in the context of graph drawing [DETT]. The general question of given a graph, can one find an embedding in the plane that minimizes the number of crossing edges, is NP-complete [GJ83]. In fact this problem is also NP-complete for a variety of special cases [SSV94]. A lot of work has been done for drawing graphs in a monotonic way in the plane. These drawings are known in the graph-drawing literature as upward planar drawings. The general problem of determining for a given directed graph, whether it can be drawn in the plane such that every edge 
is monotonically increasing in the vertical direction and no two edges cross is NP-complete, as is the problem of deciding if an undirected graph can be drawn in the plane such that every edge is a horizontal or vertical segment and no two edges cross [GT95].

In this paper we consider the following problems. Given a polygonal object (geometric graph, wire-frame or skeleton) in three dimensional euclidean space (such as a simple polygon, knot, skeleton of a Voronoi diagram or solid model mesh), we consider the problem of computing "nice" parallel (orthographic) projections of the object. If we imagine the viewer to be positioned at infinity above the $x y$-plane we are asked for a rotation of the object in space such that its projection on the $x y$-plane has the desired "niceness" properties. We consider a variety of definitions of "nice." One such definition, well known in the graph-drawing literature, is a projection with few crossings. We consider the most general polygonal object, i.e., a set of disjoint line segments. We show that given a set of $n$ line segments in space, deciding whether it admits a crossing-free projection can be done in $O\left(n^{2}\right.$ $\log n+k)$ time and $O\left(n^{2}\right)$ space, where $k$ is the number of such intersections and $k=O\left(n^{4}\right)$. This implies for example that given a simple polygon in 3-space we can determine if there exists a plane on which the projection is a simple polygon, within the same complexity. Furthermore, if such a projection does not exist, a minimum-crossing projection can be found in $O\left(n^{4}\right)$ time and $O\left(n^{2}\right)$ space. Another definition of "nice" is that of a regular projection (of interest to knot theorists) where the projection has the property that no point of the projected image corresponds to more than two points of the original object in space. We show that a set of line segments in space (which includes polygonal objects as special cases) always admits a regular projection, and that such a projection can be obtained in $O\left(n^{3}\right)$ time. A description of the set of all directions which yield regular projections can be computed in $O\left(n^{3} \log n+k\right)$ time, where $k$ is the number of intersections of a set of quadratic arcs on the direction sphere and $k=O\left(n^{6}\right)$. Finally, when the objects are polygons and trees in space, we consider monotonic projections, i.e., projections such that every path from the root of the tree to every leaf is monotonic in some direction on the projection plane. We solve a variety of such problems. For example, given a polygonal chain $P$, we can determine in $O(n)$ time if $P$ is monotonic on the projection plane, and in $O(n \log n)$ time we can find all the viewing directions with respect to which $P$ is monotonic. In addition, in $O\left(n^{2}\right)$ time, we can determine all directions for which a given tree or a given simple polygon is monotonic.

\section{Regular and Wirtinger projections}

Let $S$ be a set of $n$ distinct and disjoint line segments in $E^{3}$ specified by the cartesian coordinates of their end-points (vertices of $S$ ) and let $H$ be a plane. Let $S_{H}$ be the parallel projection of $S$ onto $H$. A parallel projection of $S$ is said to be regular if no three points of $S$ project to the same point on $H$ and no vertex of $S$ projects to the same point on $H$ as any other point on $S$ [Li93]. This definition implies that for disjoint line segments (1) no point of $S_{H}$ corresponds to more than one vertex of $S$, (2) no point of $S_{H}$ corresponds to a vertex of $S$ and an interior point of an edge of $S$, and (3) no point of $S_{H}$ corresponds to more than two interior points of edges of $S$. Therefore the only crossing points (intersections) allowed in a regular projection are those points that belong to the interiors of precisely two edges of $S$. This condition is crucial for the successful visualization and manipulation of knots [Li93]. Knots are defined as polygons in 3-D and are special cases of sets of line segments where not all segments are disjoint. Note that a vertex where two edges are joined together in the case when the line segments form a 3-D polygon counts as (not two) but one vertex. Regular projections of 3-D polygons were first studied by the knot theorist K. Reidemeister 
in 1932 [Re32] who showed that all 3-D polygons admit a regular projection and in fact almost all projections of polygons are regular. This result was re-discovered by Bhattacharya and Rosenfeld [BR94] for a restricted class of regular projections known as Wirtinger projections. Regular projections allow two consecutive edges of a 3-D polygon to project to two colinear consecutive edges on $H$. Therefore some shape features of the polygon are lost in regular projections. For visualization applications this may not be desirable. Those regular projections in which it is also required that no two consecutive edges of the 3-D polygon have colinear projections, are known as Wirtinger projections. The above authors did not address the algorithmic complexity of actually finding regular or Wirtinger projections. In this section we study the complexity of computing a single regular or Wirtinger projection as well as constructing a description of all such projections for the more general input consisting of disjoint line segments. These results include therefore results for 3-D chains, polygons, trees and geometric graphs in general. The description of all projections allows us to obtain regular or Wirtinger projections that optimize additional properties. For example, one may be interested in obtaining the most tolerant projection in the sense that it maximizes the deviation of the view-point required to violate the regularity property.

Given three line segments (edges of $S$ ) in $E^{3}$, all the directions $d$ that result in a nonregular projection of $S$ in which we have a point of $S_{H}$ that corresponds to three interior points of edges of $S$, are specified by the family of line transversals of the three edges in question. Using results by Avis and Wenger [AW87], [AW88] on transversals of three skew lines in space it can be shown that each triple of segments of $S$ yields an arc, on the unit sphere of directions, which corresponds to those directions that do not admit a regular projection. These arcs are produced by the intersections of a conic and the sphere of directions. Furthermore, these arcs have measure zero on the sphere and therefore cannot cover it. Using a similar argument it follows that the other cases also lead to measure-zero forbidden directions thus establishing the following lemma.

\section{Lemma 2.1: A set of line segments in space always admits a regular projection.}

To compute a regular projection of a set of line segments, or a description of all the directions that admit a regular projection, one may in theory compute the arrangement of the $O\left(n^{3}\right)$ arcs on the sphere. However, the intersection of two quadratic surfaces yields arcs on the sphere that are space curves of degree four and computing the arrangement of such curves is difficult in practice. A much better approach is to project these arcs from the sphere to the plane $z=1$ since then we only need to compute the arrangement of a set of quadratic arcs on the plane. There exist several optimal segment-intersection algorithms for computing the arrangement of a set of arcs on the plane. The algorithms of Chazelle \& Edelsbrunner [CE92] or Amato, Goodrich and Ramos [AGR95] do not appear to be able to be modified to handle quadratic curve segments. However, recently Balaban [Ba95] discovered an optimal algorithm that computes all intersections of quite general curves, including quadratics, that has time and space complexities $O(n \log n+k)$ and $O(n)$, respectively, where $k$ is the number of intersections among the curves. Thus we obtain the following results.

Theorem 2.2: Given a set of line segments in space, a regular projection can be obtained in $O\left(n^{3}\right)$ time. A description of the set of all directions which yield regular projections can be computed in $O\left(n^{3} \log n+k\right)$ time, where $k$ is the number of intersections of the arcs on the direction sphere and $k=O\left(n^{6}\right)$. 
One may wonder if it is worth using the optimal quadratic curve segment intersection algorithm of Balaban in practice given that there is a suboptimal but very simple algorithm due to Bentley and Ottman [BO79] that also handles quadratic curve segments and has time and space complexities $\mathrm{O}(n \log n+k \log n)$ and $\mathrm{O}(n)$, respectively, where $k$ is the number of intersections among the curves. Balaban has conducted experiments comparing his optimal algorithm to the Bentley-Ottman algorithm for as many as 4,000 segments and the latter algorithm was twice as fast. In fact, Balaban suggests that in practice the suboptimal algorithm should be used unless the number of segments is at least 200,000 .

Recall that a Wirtinger projection [BR94] of a 3-D polygon is a special type of regular projection in which no two adjacent edges project to a pair of colinear edges. We can use the above approach to compute Wirtinger projections of polygons also. For Wirtinger projections we have, in addition to the $\mathrm{O}\left(n^{3}\right)$ forbidden curve segments on the direction sphere, a set of $n$ additional forbidden great circles. Each pair of adjacent edges of the 3-D polygon yields a plane that contains them. Translate this plane to the origin and intersect it with the sphere of directions. This intersection is a forbidden great circle of directions since for each view point on this circle the two adjacent edges appear to be colinear. In total we still have $\mathrm{O}\left(n^{3}\right)$ forbidden curve segments and great circles. We therefore conclude the following.

Theorem 2.3: Given a polygon $P$ in space, a Wirtinger projection of $P$ can be obtained in $O\left(n^{3}\right)$ time. A description of the set of all directions which yield a Wirtinger projection of $P$ can be computed in $O\left(n^{3} \log n+k\right)$ time, where $k$ is the number of intersections of arcs and great circles on the direction sphere and $k=O\left(n^{6}\right)$.

\section{Minimum-crossing projections}

Whereas a regular projection of a set of line segments always exists, this is not true of crossing-free projections. To establish this it suffices to construct a counter example with three line segments very close to each other and parallel to the three orthogonal axes of the cartesian coordinate system. Here we are interested in computing a description of all the directions (if any exist) that admit crossing-free projections. Furthermore, if no crossingfree projections exist we are interested in finding projections that minimize the number of crossings. Recall that for graph-drawing problems, obtaining a minimum-crossing drawing is NP-complete [GJ83], [SSV94]. By contrast, for the projective drawing versions of these problems we provide polynomial time solutions.

Given two line segments (edges of $S$ ) in $E^{3}$, all directions $d$ that result in a non-crossing-free projection of $S$ in which we have a point of $S_{H}$ that corresponds to two points of different edges of $S$, are specified by the family of line transversals of the two edges in question. In $E^{3}$ two edges of $S$ yield a tetrahedron as a description of this family of transversals. This tetrahedron in turn determines four great-circle arcs on the unit sphere of directions that define a convex spherical quadrilateral. Thus each pair of segments of $S$ yields a spherical quadrilateral on the direction sphere that corresponds to a set of directions which results in a crossing occurring between these two line segments. Such quadrilaterals are termed forbidden. This leads to the following lemma.

Lemma 3.1: A set of disjoint line segments in space admits a crossing-free projection iff there exists a point on the sphere of directions that it is not covered by a forbidden quadrilateral. 
The set of $O\left(n^{2}\right)$ forbidden spherical quadrilaterals determined by all pairs of segments in $E^{3}$ determines a spherical arrangement on the sphere of directions. We may convert this arrangement to another arrangement of straight-line (possibly unbounded) quadrilaterals on a plane by projecting the forbidden quadrilaterals to the plane $z=1$. To determine if $S$ admits a crossing-free projection then reduces to the problem of determining if the transformed straight-line quadrilaterals cover the plane. We can do this by computing the contour of the union of these quadrilaterals. If the contour of the union is empty, then there is no direction that yields a projection without crossings.

Several algorithms have been developed for computing the contour of the union of a set of polygons. Some of these [SB92], [CN89], [NP82] are customized versions of the Bentley-Ottman line-segment intersection algorithm [BO79]. All of them compute the entire arrangement induced by the quadrilaterals and assign to each face in the arrangement, the number of quadrilaterals that intersect it. Faces numbered with zero form the contour of the union. Nievergelt \& Preparata [NP82] present a version of the algorithm tailored specifically for convex polygons whose time and space complexities are $O(n \log n+k)$ and $O(n)$, respectively, where $n$ is the number of segments in the polygons and $k$ is the number of intersections of the segments. Souvaine \& Bjorling-Sachs [SB92] propose another algorithm that computes the contour of the union from the vertical map by using topological sweep in time linear in the size of the map. This algorithm achieves the same time and space bounds as the algorithm of Nievergelt \& Preparata [NP82]. However, in order to apply either of these algorithms to compute the contour of the union in our context, they require minor modifications to handle the unboundedness of some of our quadrilaterals. Thus we have the following theorem.

Theorem 3.2: Given a set of $n$ line segments in space, deciding whether it admits a crossing-free projection can be done in $O\left(n^{2} \log n+k\right)$ time and $O\left(n^{2}\right)$ space, where $k$ is the number of edge intersections and $k=O\left(n^{4}\right)$.

If a set of line segments does not admit a crossing-free projection it is of interest to compute the projection that minimizes the number of crossings. To solve this problem we can proceed in a similar manner to that described above but this time search the entire arrangement to find the region covered with the minimum number of quadrilaterals. Therefore we have the following result.

Theorem 3.3: Given a set of $n$ line segments in space, a minimum-crossing projection can be found in $O\left(n^{4}\right)$ time and $O\left(n^{2}\right)$ space.

Besides the obvious application of minimum-crossing projections to visualization, we mention here that they also have applications to point location problems in 3-D. Consider a 3-D convex subdivision of space. Recall that the point location algorithm of Preparata \& Tamassia [PT92] projects the skeleton of the subdivision onto the xy-plane to obtain a new planar subdivision with additional vertices at all intersection points. This planar subdivision is then pre-processed for planar point location before doing binary search on the $\mathrm{z}$ direction. We can apply our algorithm to the original subdivision to minimize the memory required by the planar point location portion of their algorithm. 


\section{Monotonic projections}

The general notion of monotonicity is another characteristic of polygonal objects that aids in their visualization. A simple polygonal chain in 3-D may not admit a crossing-free projection but it may admit a projection which is monotonic in some direction. Here we are interested in determining questions such as: does a given structure admit a monotonic projection in some unspecified direction? Such problems closely resemble the NP-complete problem of determining for a given directed graph, whether it can be drawn in the plane such that every edge is monotonically increasing in the vertical direction and no two edges cross [GT95]. Again, by contrast we provide polynomial time solutions to a variety of similar orthographic projective versions of these drawing problems. First we consider the monotonicity of polygonal chains in $E^{3}$. Specifically, we address three questions. Given a polygonal chain $P$ and a direction $d$, is $P$ monotonic with respect to direction $d$ ? Recall that a polygonal chain $P=v_{1}, v_{2}, \ldots, v_{n}$ is monotonic in direction $d$ provided that the intersection of $P$ with every plane with normal $d$ is empty, or a point. We show how to answer this question in $O(n)$ time, where $n$ is the number of vertices of $P$. Next, given a polygonal chain $P$, we ask if $P$ is monotonic in some direction? We present an algorithm that determines whether a polygonal chain is monotonic in $O(n)$ time. Finally, given a polygonal chain $P$, it is of interest to determine all directions of monotonicity of $P$. We show how to compute all the directions for which $P$ is monotonic in $O(n \log n)$ time.

Given two points $a$ and $b$, let $\boldsymbol{a b}$ denote the vector directed from $a$ to $b$ and $\boldsymbol{b} a$ the vector directed from $b$ to $a$. A plane can be defined by a point $p$ contained in that plane and the normal vector $\boldsymbol{n}$ of the plane. Given a point $p$ and a vector $\boldsymbol{n}$, the plane defined by them is denoted by $H(p, n)$. Given a plane $h=H(p, n)$, we define the two half-spaces determined by this plane as follows. The open and closed half-spaces $h^{+}$are defined as $\{x / \boldsymbol{p} \boldsymbol{x} \cdot \boldsymbol{n}>0\}$ and $\{x / p \boldsymbol{x} \cdot \boldsymbol{n} \geq 0\}$, respectively. Similarly, the open and closed half-spaces $h^{-}$are defined as $\{x / p \boldsymbol{x} \cdot \boldsymbol{n}<0\}$ and $\{x / p \boldsymbol{x} \cdot \boldsymbol{n} \leq 0\}$, respectively. Henceforth, all half-spaces are open unless explicitly stated otherwise.To avoid ambiguity and simplify the discussion, we adopt the convention that if $P$ is monotonic in direction $d$, then $v_{1}$ is a minimum for $P$ with respect to $d$. We first address the question of deciding whether a polygonal chain is monotonic in a given direction. A key property of chains monotonic with respect to direction $\boldsymbol{d}$ is that their sub-chains are also monotonic with respect to $d$.

The above implies that it suffices to determine all directions for which a line segment is monotonic in order to compute all directions for which a polygonal chain is monotonic. Now a line segment is monotonic in every un-oriented direction except those perpendicular to the line segment. By our convention, we are interested in the oriented directions where line segment $[a b]$ is monotonic and $a$ is minimum with respect to the given direction. The point $a$ is a minimum with respect to all directions $D=\{\boldsymbol{d} / \boldsymbol{d} \cdot \boldsymbol{a b}>0\}$. Let $h=H(O, a b)$ (where $O$ is the origin). It follows that all directions for which $[a b]$ is monotonic can be represented by the intersection of the half-space $h^{+}$with the unit sphere $S^{2}$ that represents all directions in space (the sphere of directions). Given a polygonal chain $P=v_{1}, v_{2}, \ldots, v_{n}$ and a direction $d$, we would like to determine if $P$ is monotonic with respect to $d$. We simply verify that each of the line segments of $P:\left[v_{1}, v_{2}\right],\left[v_{2}, v_{3}\right], \ldots,\left[v_{n-1}, v_{n}\right]$ is monotonic with respect to $d$, i.e., that $\mathbf{v}_{j} \mathbf{v}_{j+1} \cdot \boldsymbol{d}>0$. We conclude with the following.

Theorem 4.1: Given a polygonal chain $P$ and a direction $d$, in $O(n)$ time, one can determine if $P$ is monotonic with respect to $d$. 
We can determine if a polygonal chain $P=v_{1}, v_{2}, \ldots, v_{n}$ is monotonic for some direction in the following way. Let $h_{i}^{+}$represent the half-space determined by the plane $H(O$, $\left.\left.v_{i} v_{i+1}\right\}\right)$. Let $D$ be the intersection of the $h_{i}^{+}$over all $i$. Then the set of all directions for which $P$ is monotonic is described by $D \cap S^{2}$. Determining if $D$, the intersection of a set of halfspaces is non-empty can be accomplished in linear time using linear programming [Me83]. Therefore we conclude with the following.

Theorem 4.2: Given a polygonal chain $P$, one can determine if $P$ is monotonic in $O(n)$ time.

As noted above, $D \cap S^{2}$ describes the set of all the directions from which $P$ is monotonic. Since the intersection of a set of half-spaces can be computed in $O(n \log n)$ time [PS85], we conclude with the following.

Theorem 4.3: Given a polygonal chain $P$, one can determine in $O(n \log n)$ time all the directions with respect to which $P$ is monotonic.

Now we turn to the monotonicity of simple polygons and trees in $E^{3}$.The polygonal chains, simple polygons and trees in $E^{3}$ are all graphs embedded in $E^{3}$. In order to continue the discussion in this more general setting, we define a geometric graph. A geometric graph is a two-tuple $(V, E)$, where $V$ is a finite set of distinct points in general position in $E^{3}$, and $E$ is a family of closed straight-line segments with end-points in $V$. The elements of $V$ and $E$ are called vertices and edges, respectively. For more definitions and terminology concerning graphs, the reader is referred to [BM76]. In the previous section, the geometric graphs that we considered were paths. In this section, we concentrate on trees and cycles (polygons). We begin by describing some properties of geometric graphs. Given a vertex $v$ of a geometric graph $G$, we denote the set of $e$ dges adjacent to $v$ by $E A(v)$.

Lemma 4.4: Vertex $v$ is a minimum with respect to $d$ for $E A(v)$ if and only if $\forall e \in E A(v)$, $v$ is a minimum with respect to $d$ for $e$.

Given a vertex $v$ of a geometric graph $G$, we denote by $M D(v)$ the set of directions for which $v$ is a minimum for the set $E A(v)$. Let $e=\left[v v_{i}\right]$ be an edge in $E A(v)$. By $e$ we denote the vector $v v_{i}$. Let $h(e)=H(O, e)$. We see that $M D(v)$ is the intersection of $h^{+}(e)$ over all $e$ contained in $E A(v)$. A vertex $v$ of a geometric graph is a proper local minimum with respect to direction $d$ provided that $v$ is a minimum for the set $E A(v)$ in direction $d$. A vertex $v$ is a local minimum with respect to direction $d$ if $\forall e \in E A(v)$, the edge $e$ is contained in the closure of $H^{+}(\nu, d)$.

We now address several questions concerning the monotonicity of trees. Suppose we are given a rooted tree $T$, and a direction $d$. The first question we address is to determine if $T$ is monotonic in direction $\boldsymbol{d}$. Notice that two things are specified in this question, the root of the tree and the proposed direction of monotonicity. The next four questions we address are the following: (1) Given a rooted tree $T$, does there exist a direction $d$ for which $T$ is monotonic? (2) Given an unrooted tree $T$ and direction $d$, does there exist a root such that $T$ is monotonic with respect to $d$ ? (3) Given an unrooted tree $T$, does there exist a direction $d$ and a root of $T$ such that $T$ is monotonic with respect $d$ ? (4) Given an unrooted tree $T$, find all roots and directions for which $T$ is monotonic. 
Recall that a tree $T$ is called a rooted tree if a unique vertex $v$ of $T$ is specified to be the root, otherwise the tree is unrooted or free. A rooted tree $T$ is monotonic in direction $d$ provided that the path from the root to every vertex is monotonic in direction $d$. The key behind the efficient solution of all above-mentioned problems depends on the following characterization of the monotonicity of rooted trees.

Lemma 4.5: A rooted tree $T$ is monotonic in direction $d$ if and only if the root $r$ of $T$ is $a$ proper local minimum and no other vertex is a local minimum with respect to direction $d$.

Proof: $\quad \Rightarrow$ Assume $T$ is monotonic with respect to $d$. If $r$ is not a proper local minimum then at least one root to leaf path in $T$ is not monotonic. Suppose there exists a vertex $v$ of $T$ that is not the root, such that $v$ is a local minimum. Let $h=H(v, d)$. We see that $E A(v)$ must be in the closure of $h^{+}$. Let $P$ be the unique path from $r$ to $v$ in $T$. $P$ must be monotonic since $T$ is monotonic. Also, the root $r$ is a minimum and $v$ is a maximum for $P$ with respect to direction $d$, by the convention of monotonic paths. Therefore, $P v$ must be contained in $h^{-}$. Let $v_{j}$ be the vertex preceding $v$ in $P$. Since $v_{j}$ is adjacent to $v$, we see that $\left[v v_{j}\right] \in E A(v)$. But this implies that $\left[v v_{j}\right]$ is contained in the closure of $h^{+}$which contradicts the monotonicity of $P$.

$\Leftrightarrow$ Given a vertex $v$, we will represent the plane $H(v, d)$ by $h_{\mathrm{v}}$. Assume that the root $r$ of $T$ is the only proper local minimum and no other vertex of $T$ is a local minimum.

Let $v$ be an arbitrary vertex of $T$. We will show that the path $P$ from $r$ to $v$ must be monotonic in direction $d$. Let the path $P=v_{1}(=r), v_{2}, \ldots, v_{k-1}, v_{k}(=v)$. Suppose $P$ is not monotonic with respect to $d$. Let $v_{i}$ be the first vertex of $P$ such that $\left[v_{i-1} v_{i}\right.$ ] is not monotonic with respect to $d$. Since $\left[v_{i-1} v_{i}\right.$ ] is not monotonic with respect to $d$, we conclude that $v_{i}$ must be contained in the closure of $h_{v_{j}}$. Since $v_{i}$ is not a local minimum, and $T$ is acyclic, there must exist a vertex $v_{j}$ in $E A\left(v_{i}\right)$ different from $v_{i-1}$, such that $v_{j}$ is contained in $h_{v_{i}}$. Similarly, since $v_{j}$ is not a local minimum, there must exist a $v_{m}$ in $E A\left(v_{j}\right)$ different from $v_{j}$, such that $v_{m}$ is contained in $h_{v_{j}}$. By continuing this argument, it follows that since there are only a finite number of vertices in $T$, there must be a vertex $v_{t}$ such that $E A\left(v_{t}\right)$ is contained in the closure of $h_{v_{t}}{ }^{+}$, contradicting the fact that no vertex of $T$ is a local minimum.

Suppose we are given a tree $T$, a root $r$ of $T$ and a direction $d$ and want to determine whether $T$ is monotonic with respect to $d$. By Lemma 4.5 , since $r$ is the only proper local minimum and no other vertex is a local minimum with respect to $d$, the direction $\boldsymbol{d}$ cannot be contained in the closure of $M D(v)$ for all vertices of $T$ other than the root. Since $M D(v)$ is an intersection of half-spaces, determining whether or not a direction $\boldsymbol{d}$ is contained in $M D(v)$ can be done in $O(|M(v)|)$ time. But $O(|M(v)|)$ is $O(d(v))$ where $d(v)$ is the degree of $\nu$ in the tree $T$. Therefore, since the sum of the degrees of the vertices of a tree is linear in the number of vertices of the tree, we conclude that in $O(n)$ time where $n$ is the number of vertices of $T$, we can determine if a rooted tree is monotonic in a given direction $d$.

Theorem 4.6: Given a rooted tree $T$, and a direction d, one can decide in $O(n)$ time if $T$ is monotonic with respect to $d$.

Suppose next that the root of the tree is no longer specified. Then, to determine if $T$ is monotonic in direction $d$, we must first find a root. By Lemma 4.5 , the root must be the only proper local minimum and no other vertex is a local minimum. Therefore, there must 
exist exactly one vertex $r$ of $T$ such that $d$ is contained in $M D(r)$, which becomes the root. All other vertices $v$ of $T$ must have the property that $d$ is not in the closure of $M D(v)$. Again this can be determined in linear time. Therefore, we conclude with the following.

Theorem 4.7: Given an unrooted tree $T$, and a direction d, one can decide in $O(n)$ time where $n$ is the number of vertices of $T$, if there exists a root $r$ of $T$ such that $T$ is monotonic with respect to $d$.

Before continuing, we need a few more preliminaries. Instead of representing all directions in 3-space by the sphere of directions $S^{2}$, we will represent all directions in $E^{3}$ by points on the surface of the axis-parallel cube $A C$ centered at the origin $O$ and with edge length 2. A point $p$ on $A C$ represents the direction $O p$. Although this representation is not standard, it will simplify many of the algorithms to follow.

Now, suppose that the root $r$ of $T$ is specified but the direction is not. For $T$ to be monotonic in some direction $d$, we see that $d$ must be in $M D(r)$ but outside $M D(v)$ for all vertices $v$ of $T$. This problem is in fact as difficult as the general problem where given an unrooted tree $T$, find all possible roots and directions for which $T$ is monotonic. As such we will present the solution to the general problem below.

The intersection of $M D(v)$ and $A C$ represents the set of directions for which $v$ is a proper local minimum. Since $M D(v)$ is the intersection of a set of half-spaces, the intersection of $M D(v)$ with a facet $F$ of $A C$ is either empty, the facet itself, or a convex polygon. For each facet $F_{i}$ of $A C(1 \leq i \leq 6)$ and each vertex $v_{j}$ of $T(1 \leq j \leq n)$, we compute the intersection $I(i, j)=M D\left(v_{j}\right) \cap F_{i}$. On each facet $F_{i}$, notice that the set $I_{i}=\{I(i, j) / 1 \leq j \leq n\}$ is simply a collection of convex polygons. This collection of polygons has the following property. If a point $p \in F_{i}$ is contained in the interior of $k$ polygons of the set $I_{i}$, then there are $k$ vertices of $T$ that are local minima with respect to the direction $O p$ and each of the vertices that are proper local minima is identified by the polygon which contains $p$. That is, if $p$ is contained in polygon $I(i, 3)$ then vertex $v_{3}$ is a proper local minimum with respect to direction $O p$. Therefore, to determine if there are any directions with respect to which $T$ is monotonic, we want to determine if there are any regions in each facet $F_{i}(1 \leq i \leq 6)$ that are covered by only one polygon of the set $I_{i}(1 \leq i \leq 6)$. In fact, we want to find all regions that are covered by only one polygon. This set of regions represents the set of all the directions and roots from which $T$ is monotonic.

Let $A_{i}$ represent the subdivision induced on facet $F_{i}$ by the set of polygons $I_{i}$. This subdivision can be computed deterministically in $O(n \log n+t)$ time where $t$ is the total number of intersection points of all polygons in $I_{i}$. The complexity of $A_{i}$ is $O\left(n^{2}\right)$. Consider the graph $G_{i}$ which has a node for every cell of $A_{i}$, and an edge between two nodes if the corresponding cells are incident to the same edge of $A_{i}$. The graph $G_{i}$ has $O\left(n^{2}\right)$ nodes and edges. Start at any node $a_{1}$ of $G_{i}$ and compute in $O(n)$ time how many polygons of $I_{i}$ cover it. Store this number with $a_{1}$. Start from $a_{1}$ with a depth first search. Every edge $\left(a_{1}, a_{m}\right)$ of $G_{i}$ we traverse corresponds to going inside or outside a polygon of $I_{i}$, in which case we take the number of $a_{1}$ and add or subtract one from it and assign this number to $a_{m}$. Thus the whole process of assigning values to nodes of $G_{i}$ can be done in $O\left(n^{2}\right)$ time. Let $M_{i}$ represent the set of nodes with the minimum number assigned to it. If this number is one, then each of the cells represented by a node in $M_{i}$ represents a set of directions and a root from which $T$ is monotonic. The root of $T$ is specified by the vertex generating the convex polygon covering the cell. 
Theorem 4.8: In $O\left(n^{2}\right)$ time, we can determine all directions for which $T$ is monotonic.

Consider now the problem of determining the monotonicity of a simple polygon in $E^{3}$. We begin with a few definitions. A simple polygon in $E^{3}$ is a geometric graph that is a cycle. A simple polygon $P$ is monotonic in direction $d$ provided there exist two vertices $u, v$ of $P$ such that both paths from $u$ to $v$ are monotonic in direction $d$.

The characterization of monotonicity of simple polygons in $E^{3}$ is similar to that of trees and therefore the solution for trees is applicable in this case. Therefore, we conclude with the following.

Theorem 4.9: Given a simple polygon $P$ in $E^{3}$ and a direction $d$, in $O(n)$ time it can be determined if $P$ is monotonic with respect to $d$.

Theorem 4.10: Given a simple polygon $P$ in $E^{3}$, in $O\left(n^{2}\right)$ time, we can determine all the directions with respect to which $P$ is monotonic.

\section{Conclusion}

Our results on regular and minimum-crossing projections of line segments have immediate corollaries for polygonal chains, polygons, trees and more general geometric graphs in 3-D since these are all special cases of sets of line segments. Our resuits also have application to graph drawing for knot-theorists. Let $K$ be a knot with $n$ vertices. To study the knot's combinatorial properties, knot theorists obtain a planar graph $G$ called the diagram of $K$ by a regular projection of $K$. Many of their algorithms are applied to $G$ and therefore their time complexity depends on the space complexity of $G$. By combining our algorithms we can obtain regular projections with the minimum number of crossings thereby minimizing the time complexity of their algorithms.

\section{Acknowledgments}

The authors thank Suneeta Ramaswami, Fady Habra, Ivan Balaban, Bernard Chazelle and Mike Goodrich for correspondence. The second author thanks Lola for her support.

\section{References}

[AGR95] Amato, N. M., Goodrich, M. T. and Ramos, E. A., "Computing faces in segment and simplex arrangements," Proc. Symp. on the Theory of Computing, 1995.

[AW88] Avis, D., and Wenger, R., "Polyhedral line transversals in space," Discrete and Computational Geometry, vol. 3, 1988, pp. 257-265.

[AW87] Avis, D., and Wenger, R., "Algorithms for line stabbers in space," Proc. Third ACM Symp. on Computational Geometry, 1987, pp. 300-307.

[Ba95] Balaban I. J., "An optimal algorithm for finding segments intersections," Proc. ACM Symp. on Comp. Geom., Vancouver, Canada, June 1995, pp. 211-219.

[BO79] Bentley, J.L. and Ottmann, T.A., "Algorithms for reporting and counting geometric intersections", IEEE Trans. Comput. vol. 8, pp. 643-647, 1979.

[BR94] Bhattacharya, P. and Rosenfeld, A., "Polygons in three dimensions," J. of Visual Communication and Image Representation, vol. 5, June 1994, pp. 139-147.

[BM76] Bondy, J. and Murty, U. S. R., Graph Theory with Applications, Elsevier Science, New York, 1976.

[BGK95] Burger, T., Gritzmann, P. and Klee, V., "Polytope projection and projection polytopes," TR No. 95-14, Dept. Mathematics, Trier University. 
[CE92] Chazelle, B. and Edelsbrunner, H., "An optimal algorithm for intersecting line segments in the plane," J. ACM, vol. 39, 1992, pp. 1-54.

[CN89] Chiang, K., Nahar, S., and Lo, C., "Time-efficient VLSI artwork analysis algorithms in GOALIE2", IEE Trans. CAD, vol. 39, pp. 640-647, 1989.

[Co90] Colin, C., "Automatic computation of a scene's good views," MICAD'90, Paris, February, 1990.

[DETT] Di Battista, G., Eades, P., Tamassia, R. and Tollis, I. G., "Algorithms for drawing graphs: an annotated bibliography," Computational Geometry: Theory and Applications, vol. 4, 1994, pp. 235-282.

[FDFH] Foley, J. D., van Dam, A., Feiner, S. K. and Hughes, J. F., Computer Graphics: Principles and Practice, Addison-Wesley, 1990.

[Ga95] Gallagher, R. S., Ed., Computer Visualization: Graphic Techniques for Engineering and Scientific Analysis, IEEE Computer Society Press, 1995.

[GJ83] Garey, M.R., and Johnson, D.S., "Crossing number is NP-complete," SIAM J. Alg. Discrete Methods, vol. 4, 1983, pp. 312-316.

[GT95] Garg, A. and Tamassia, R., "On the computational complexity of upward and rectilinear planarity testing," eds., R. Tamassia and I. G. Tollis, Proc. Graph Drawing'94, LNCS 894, Springer-Verlag, 1995, 286-297.

[HMTT] Hirata, T., Matousek, J., Tan, X.-H. and Tokuyama, T., "Complexity of projected images of convex subdivisions," Comp. Geom., vol. 4., 1994, pp. 293-308.

[KK88] Kamada, T. and Kawai, S., "A simple method for computing general position in displaying three-dimensional objects," Computer Vision, Graphics and Image Processing, vol. 41, 1988, pp. 43-56.

[KK93] Keller, P. R. \& Keller, M. M., Visual Cues: Practical Data Visualization, IEEE Computer Society Press, 1993.

[Li93] Livingston, C., Knot Theory, The Carus Mathematical Monographs, vol. 24, The Mathematical Association of America, 1993.

[Me83] Megiddo, N., "Linear-time algorithms for linear programming in $R^{3}$ and related problems," SIAM Journal of Computing, vol. 12, 1983, pp. 759-776.

[MS85] McKenna, M. \& Seidel, R., "Finding the optimal shadows of a convex polytope," Proc. ACM Symp. on Comp. Geom., June 1985, pp. 24-28.

[NP82] Nievergelt, J., Preparata, F., "Plane-sweep algorithms for intersecting geometric figures", Communications of ACM vol.25, pp. 739-747, 1982.

[PS85] Preparata, F., and Shamos, M., Computational Geometry: An introduction, Springer-Verlag, New York, 1985.

[PT92] Preparata, F. and Tamassia, R., "Efficient point location in a convex spatial cellcomplex," SIAM Journal of Computing, vol. 21, 1992, pp. 267-280.

[Re83] Reidemeister, R., Knotentheorie, Ergebnisse der Mathematic, Vol. 1, SpringerVerlag, Berlin, 1932; L. F. Boron, C. O. Christenson and B. A. Smith (English translation) Knot Theory, BSC Associates, Moscow, Idaho, USA, 1983.

[SB92] Souvaine, D. and Bjorling-Sachs, I., "The contour problem for restricted-orientation polygons", Proc. of the IEEE, vol. 80, pp. 1449-1470, 1992.

[SSV94] Shahrokhi, F., Szekely, L., and Vrt'o, I., "Crossing number of graphs, lower bound techniques and algorithms: A survey," Lecture Notes in Comp. Science, vol. 894, Princeton, New Jersey, 1994, pp. 131-142.

[St82] Strang, G., "The width of a chair," The American Mathematical Monthly, vol. 89, No. 8, October 1982, pp. 529-534.

[To85] Toussaint, G. T., "Movable separability of sets," in Computational Geometry, G. T. Toussaint, Ed., Elsevier Science Publishers, 1985, pp. 335-375. 